\title{
Laboratório Digital à Distância: Percepções de Docentes e Discentes
}

\author{
Felipe Valencia de Almeida \\ felipe.valencia.almeida@usp.br \\ Universidade de São Paulo \\ São Paulo, Brasil
Edson Toshimi Midorikawa emidorik@usp.br \\ Universidade de São Paulo \\ São Paulo, Brasil
}

\author{
Victor Takashi Hayashi \\ victor.hayashi@usp.br \\ Universidade de São Paulo \\ São Paulo, Brasil \\ Paulo Sergio Cugnasca \\ cugnasca@usp.br \\ Universidade de São Paulo \\ São Paulo, Brasil
}

\author{
Reginaldo Arakaki \\ reginaldo.arakaki@poli.usp.br \\ Universidade de São Paulo \\ São Paulo, Brasil \\ Sergio Roberto de Mello \\ Canovas \\ sergio.canovas@usp.br \\ Universidade de São Paulo \\ São Paulo, Brasil
}

\begin{abstract}
RESUMO
O período corrente de isolamento social, adotado como medida para conter a pandemia do COVID-19, deixou aparente a necessidade de repensar métodos e técnicas de ensino, visando assegurar o aprendizado tanto teórico quanto prático dos discentes num momento em que o aprendizado presencial está inviabilizado. Este artigo apresenta a percepção docente e discente, que foram obtidas através de questionários realizados em workshops com os mesmos, de um oferecimento à distância de uma disciplina de laboratório de eletrônica digital em andamento. O oferecimento da disciplina é viabilizado por meio de uma infraestrutura desenvolvida utilizando conceitos de Internet das Coisas, permitindo o oferecimento de uma disciplina prática e respeitando as regras impostas de distanciamento social. Os resultados favoráveis obtidos até o momento foram possíveis através de um trabalho conjunto entre professores, monitores, técnicos e alunos.
\end{abstract}

\section{CCS CONCEPTS}

- General and reference $\rightarrow$ Experimentation; $\bullet$ Computer systems organization $\rightarrow$ Distributed architectures.

\section{PALAVRAS-CHAVE}

laboratório de eletrônica digital, laboratório remoto, fpga

\section{INTRODUÇÃO}

Disciplinas de laboratório de eletrônica digital, também chamadas de Laboratório Digital, utilizam ferramentas de implementação, síntese e simulação de circuitos digitais com linguagens de descrição de hardware, como o VHDL (VHSIC Hardware Description Language) por exemplo. As simulações são ferramentas essenciais para o projeto de circuitos digitais. Entretanto, uma limitação à aprendizagem é que simulações não conseguem proporcionar a sensação de execução presente em um sistema real [6]. Além disso,

Fica permitido ao(s) autor(es) ou a terceiros a reprodução ou distribuição, em parte ou no todo, do material extraído dessa obra, de forma verbatim, adaptada ou remixada, bem como a criação ou produção a partir do conteúdo dessa obra, para fins não comerciais, desde que sejam atribuídos os devidos créditos à criação original, sob os termos da licença CC BY-NC 4.0.

EduComp'21, Abril 27-30, 2021, Jataí, Goiás, Brasil (On-line)

(c) 2021 Copyright mantido pelo(s) autor(es). Direitos de publicação licenciados à Sociedade Brasileira de Computação (SBC). a execução permite detectar problemas que a simulação por sua vez não permitiria. Estas ferramentas existentes de simulação também possuem limitações em relação ao nível de interação possível por parte dos usuários [9]. Além das aulas teóricas presentes na educação à distância, o uso de laboratório para a Engenharia é um aspecto importante para o aprendizado dos alunos [13].

Com a pandemia do coronavírus (Covid-19), a execução de disciplinas práticas de Engenharia enfrenta problemas adicionais no contexto do distanciamento social implementado em instituições de ensino superior. A suspensão temporária destas disciplinas práticas ou então a reestruturação das mesmas, sem o contato direto com o aparato experimental, têm sido medidas adotadas como forma de contornar os efeitos da crise, de maneira a viabilizar a reestruturação das disciplinas. O problema destas medidas de caráter emergencial é o impacto tanto no aprendizado do aluno quanto em sua capacidade de relacionar a teoria vista nas disciplinas teóricas com a prática do mundo real. Estes fatores são fundamentais para manter o engajamento do aluno no curso, o que pode reduzir a taxa de evasão do mesmo. Desta forma, o desenvolvimento e compartilhamento de ferramentas que tornem possível a realização de experimentos de forma remota pode ajudar neste cenário.

Dentre as vantagens da abordagem de laboratório remoto com FPGA (Field Programmable Gate Array), destacam-se custos reduzidos, maior flexibilidade, e potencial para melhor utilização dos recursos disponíveis no laboratório [7]. Um relato presente na literatura descreve que, entre os dispositivos disponíveis nas duas modalidades (laboratório remoto e laboratório presencial), os estudantes utilizaram muito mais os equipamentos por meio de laboratório remoto do que no laboratório presencial [12]. Segundo [13], o laboratório remoto permite maiores oportunidades de aprendizado aos alunos, a partir da ampliação de horários para uso do laboratório.

A teoria de estilos de aprendizagem pode ajudar a entender como alunos percebem, interagem e aprendem de formas diferentes. Esta teoria disserta sobre como aspectos cognitivos, culturais, fisiológicos, culturais e psicológicos influenciam em estratégias de aprendizado por pessoas. Barros [1] apresenta quatro estilos principais: ativo, reflexivo, teórico e pragmático. Alunos do estilo ativo gostam de novas experiências, enquanto alunos do estilo reflexivo gostam de analisar sob diversas perspectivas de forma prudente, alunos do estilo teórico se adaptam bem a teorias lógicas, e alunos do estilo 
pragmático são experimentadores, práticos, e buscam aproveitar a primeira oportunidade de aplicar novas ideias.

A oportunidade identificada a partir do exposto é a proposição de uma ferramenta de laboratório digital remoto, que complementa as simulações realizadas por alunos em seus computadores pessoais, para apoio às atividades práticas da disciplina de laboratório de eletrônica digital durante a pandemia do coronavírus (Covid-19).

É esperado que a ferramenta ajude alunos do estilo de aprendizagem pragmático a aprender na prática conceitos de projeto e desenvolvimento de sistemas digitais, e que alunos de outros estilos de aprendizagem possam complementar suas formas de aprender [1]. São apresentados a seguir o repositório com todo o código-fonte do projeto (licença creative commons), e um conjunto de vídeos com demonstrações de experimentos realizados.

Vídeos de demonstração na plataforma Youtube.

Código Aberto em Repositório na plataforma Github.

\section{TRABALHOS RELACIONADOS}

Na literatura foram encontrados alguns trabalhos relacionados envolvendo a estruturação de um laboratório remoto para ensino de projeto de circuitos digitais com placas FPGA.

O iLabs Logic Design iLab do MIT utiliza uma placa FPGA Altera DE1 e permite a síntese de circuitos descritos em VHDL [3]. Seus principais objetivos foram incentivar o uso de laboratórios remotos (denominados iLabs) no ensino de Ciências e Engenharia e elaborar um framework para o desenvolvimento e compartilhamento de iLabs. O projeto iLab do MIT é documentado em [10].

O laboratório remoto RFL (Remote FPGA Lab) suporta controle e monitoramento de placas FPGA. Interações são realizadas através de interfaces visuais, em uma arquitetura de computação em nuvem. Foi utilizado para ensino à distância, suporte a demonstrações e uso por estudantes fora do horário de aula [9].

Outra solução presente na literatura permite que o usuário forneça o arquivo com o circuito descrito em VHDL, e a automação de diversas etapas como compilação e mapeamento de pinos. Utiliza placas FPGA Altera DE2 e software Altera Quartus, e os testes são realizados a partir do arquivo de testes fornecido pelo usuário [6].

Uma ferramenta para o ambiente da empresa Xilinx permite acesso remoto a placas FPGA. É baseada no sistema operacional Ubuntu, servidor Apache, e foi desenvolvido em PHP e Python. A arquitetura é baseada na computação em nuvem, e integra interfaces de linha de comando (CLI, do inglês command line interface) e gráfica (GUI, do inglês graphical user interface) [4].

O laboratório remoto descrito em [13] permite que o aluno realize a descrição e simulação do circuito digital em seu computador, e faça o upload do arquivo de programação da FPGA em um servidor remoto. Este servidor é responsável por realizar a carga, obter os resultados do experimento após aproximadamente 1 minuto e enviálos de volta ao aluno [13].

A Universidade do Cairo no Egito possui um projeto de laboratório remoto com FPGA, com interfaces de linha de comando e gráfica para controlar e monitorar sinais da placa em tempo real. Utiliza o sistema operacional Linux, diversas ferramentas para acesso remoto (e.g., desktop remoto), servidor Apache e possui interface web. Os dispositivos físicos que integram a solução são um servidor, $\mathrm{e}$ placas FPGA equipadas com uma câmera cada [8].
A partir do laboratório remoto WebLab-Deusto, uma solução com foco em usabilidade foi desenvolvida para suporte a uma disciplina de modelagem digital. Possui vídeos de suporte para permitir o aprendizado à distância. Como um fator de sucesso para abordagens de laboratório remoto, esta solução foi especificada a partir da perspectiva dos alunos [11].

Pelo exposto, apesar da ideia de laboratório remoto com placa FPGA não ser inédita, todas as soluções pesquisadas oferecem exclusivamente interface desktop para os alunos. O diferencial deste trabalho para o ensino utilizando um laboratório remoto é que ele permite compilação, carga, mapeamento de pinos e interação com placas FPGA por meio de uma interface mobile (i.e. acessível por tablets e smartphones), com o uso de tecnologias de Internet das Coisas.

\section{APRESENTAÇÃO DA PLATAFORMA}

A plataforma é composta por duas partes: a bancada remota, localizada fisicamente no laboratório e o aluno na sua residência, conforme ilustrado pela Figura 1. No contexto deste trabalho, o termo "plataforma"é utilizado para designar uma infraestrutura de apoio projetada que viabiliza o oferecimento de uma disciplina prática de eletrônica digital de maneira remota. Esta infraestrutura é composta tanto por dispositivos eletrônicos quanto por ferramentas computacionais. Para descrevê-la, inicialmente será feita uma apresentação da plataforma IoT, para em seguida apresentar a função de cada um dos dispositivos eletrônicos conectados.

Destaca-se na figura o conceito de LabHome, que representa um laboratório na própria residência do aluno. Mesmo que sua implementação não seja parte o escopo deste artigo, é importante destacar sua importância no contexto global do projeto. Com o LabHome, o aluno pode integrar componentes eletrônicos em sua própria residência com os componentes disponíveis no laboratório de maneira remota, por meio de um segundo dispositivo ESP8266, expandindo as possibilidades de abordar conteúdos e experiências distintas.

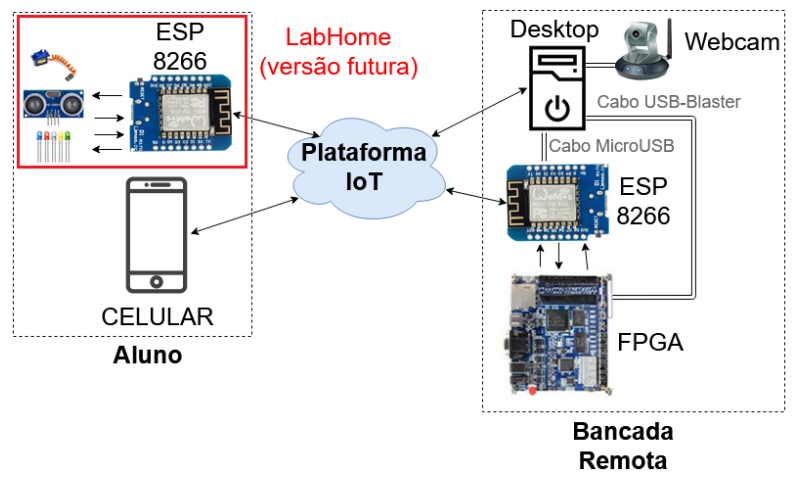

Figura 1: Arquitetura da plataforma

Em relação aos requisitos mínimos de hardware, os requisitos do celular estão relacionados a plataforma IoT escolhida, já os requisitos do desktop ao ambiente de desenvolvimento utilizado para programar as placas FPGAs, que é o software com maior custo computacional. Neste cenário apresentado a seguir, com a definição das 
partes da plataforma, é necessário que o celular do aluno possua o sistema operacional Android 4.2/iOS 9.0 (ou versões superiores), já o desktop localizado no laboratório necessita de pelo menos $15 \mathrm{~GB}$ de armazenamento, 4 GB de memória RAM e sistema operacional Windows 7 (ou versões superiores) com a possibilidade de utilizar distribuições Linux. Para a instalação do projeto no laboratório, outros componentes são necessários: placa de desenvolvimento Wemos do ESP8266, cabos de conexão, placas protoboard, cabos USB para compilação e carga do ESP8266, webcam, módulos expansores de portas I2C (por exemplo, PCF8574) e componentes específicos de cada experimento (por exemplo, servomotores ou sensores ultrassônicos). Também é necessário que o laboratório possua uma rede local WiFi $802.112,4 \mathrm{GHz}$ com Internet.

\subsection{Plataforma IoT e Celular}

A plataforma IoT escolhida para o projeto da plataforma foi o Blynk. Blynk [2] consiste em uma plataforma de computação em nuvem que efetua associação via Internet entre sinais de entrada/saída digital de dispositivos eletrônicos e interfaces mobile. Através do paradigma de pinos virtuais que podem ser mapeados a pinos físicos de dispositivos embarcados, e pinos virtuais presentes em aplicações mobile, é possível conectar dispositivos de forma transparente, sem que os usuários tenham de se preocupar com aspectos de infraestrutura de comunicação. Para utilizá-lo é necessário instalar um aplicativo gratuito para dispositivos Android ou iOS. Após isso, é necessário criar uma conta (gratuita ou paga) para usar essa plataforma. No contexto da plataforma proposta, a conta gratuita supre as necessidades de uso.

No Blynk, é possível criar projetos, onde cada projeto é feito especificamente para algum componente digital. Após a criação de um projeto, um token de autenticação é gerado, sendo que o compartilhamento deste token é necessário para estabelecer a comunicação entre os diversos componentes utilizados na plataforma.

No projeto pode-se instanciar uma série de componentes no Blynk, chamados de Widgets, como botões, leds, terminais, displays, etc. Cada Widget do Blynk gera sinais digitais virtuais que podem ser mapeados nos pinos físicos de dispositivos IoT que executam bibliotecas do Blynk. Desta forma, é feita uma conexão entre dispositivos físicos e aplicativos. O uso de algum desses componentes no projeto consome "pontos de energia"do Blynk, nome comercial dado aos créditos da plataforma. Ou seja, o limite de widgets que o usuário pode adicionar a um projeto Blynk é determinado pela quantidade de "pontos de energia"que ele possui, o que depende de a conta ser gratuita ou paga. A Figura 2 apresenta um exemplo de projeto no Blynk, onde foram instanciados um terminal, três botões e três leds. É importante ressaltar que no momento em que esses componentes são instanciados no projeto, eles são acessíveis tanto no próprio aplicativo quanto no servidor em nuvem do Blynk via API (Application Programming Interface). Desta forma, é possível realizar tanto a leitura quando alterar o valor desses componentes através de requisições HTTP. Para o uso da plataforma, é necessário apenas a instanciação de um terminal virtual no projeto do Blynk.

\subsection{Placa FPGA}

A placa FPGA é a base da disciplina oferecida de laboratório de eletrônica digital. Nela, é possível sintetizar o comportamento de

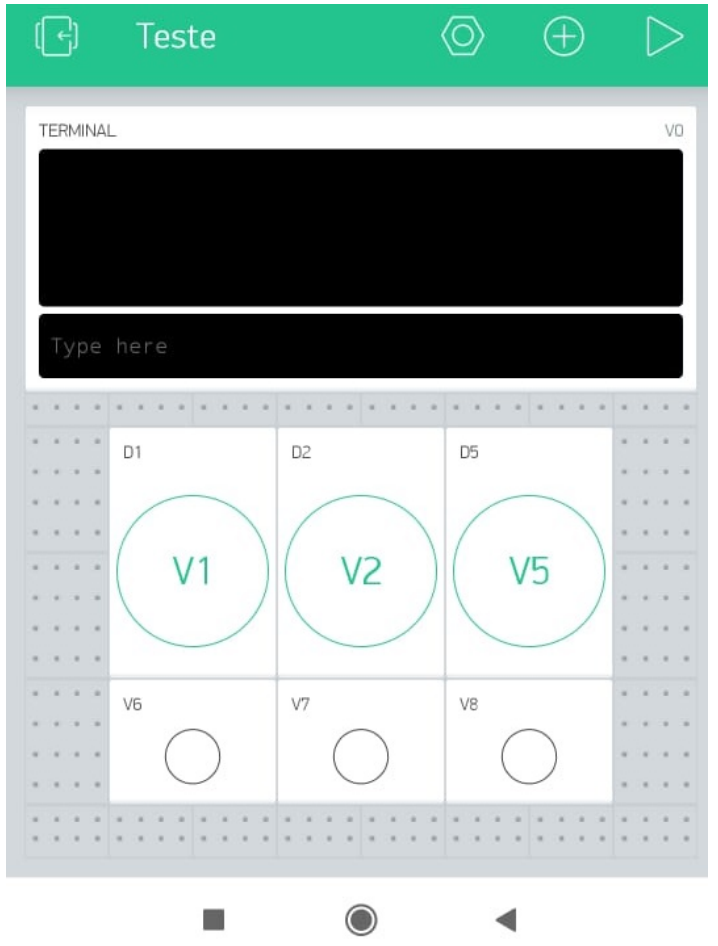

Figura 2: Tela de um projeto criado no Blynk

circuitos digitais através da carga de um fluxo de bits (bitstream) gerado a partir da descrição de algum circuito com uma linguagem de descrição de hardware, como o VHDL. Em um oferecimento convencional da disciplina, o aluno realiza todo o procedimento de compilação do circuito, carga na placa FPGA e testes de forma presencial acionando botões e chaves, pelo fato de estar localizado fisicamente próximo da placa. A função da plataforma é permitir que esses processos sejam realizados de maneira remota, fornecendo um suporte para tal.

A versão atual da plataforma aqui apresentada foi projetada de tal forma a suportar placas FPGAs distintas da fabricante Intel (antiga Altera), sendo que a plataforma pode ser adaptada para utilizar FPGAs de outros fabricantes. Para o oferecimento da disciplina é utilizada a placa DE0-CV [5], juntamente com o software Quartus Prime, que é necessário para gerar o arquivo de programação (bitstream) específico para essa placa.

\subsection{ESP8266}

O ESP8266 é o agente remoto que efetua as interações na FPGA equivalentes às que o aluno faria presencialmente, tal como alterar o estado de uma chave ou botão, recebendo comandos remotamente pela nuvem do Blynk. Desta forma, conforme foi apresentado na Figura 1, o ESP8266 localizado na bancada remota está conectado com a placa FPGA para viabilizar a troca de sinais de entrada/saída, e conectado à Internet. Para tal, foi utilizada uma placa Wemos D1 mini, que contém o ESP8266. Ressalta-se aqui que a escolha desta placa e do próprio ESP8266 foi dada em função de seu custobenefício e possibilidade de ser utilizado de forma independente (i.e. 
standalone) com a plataforma Blynk. Diversos outros componentes poderiam ser utilizados com o mesmo propósito, como o Arduino Uno com um módulo Wi-Fi externo, ou o Rasberry Pi, porém com maior custo associado. A conexão é feita ligando os pinos da placa Wemos D1 mini com os pinos GPIO (General Purpose Input/Output) da placa FPGA.

O módulo ESP8266 então é carregado com um script feito com a própria IDE do Arduino que permite a leitura de dados de um terminal do Blynk, através de requisição HTTP. Com base no que é lido, ele pode alterar o valor de alguma entrada da placa FPGA ou então ler alguma saída da mesma, e apresentar ao usuário por meio do envio dos resultados ao terminal virtual.

\subsection{Desktop}

O desktop é a máquina remota disponível no laboratório físico da disciplina. Ela executa uma série de scripts Python para coordenar a comunicação do aluno com a bancada remota e executar comandos de síntese do projeto VHDL e acionamento de sinais de teste para a placa FPGA. Há um cabo USB conectando o computador com a FPGA, que permite o envio dos comandos necessários pelo script. A coordenação é feita através de operações de leitura e escrita do terminal do Blynk com requisições HTTP. Desta forma, o desktop se comunica indiretamente com o celular do aluno, por intermédio do servidor na nuvem do Blynk, que armazena os dados do terminal. O fato de não existir uma comunicação direta entre o celular do aluno e a máquina remota contribui em garantir o requisito de segurança do sistema, além de facilitar a configuração e setup, pois não é necessário a abertura de portas no roteador do laboratório ou do aluno.

\subsection{Webcam}

A função da webcam é fornecer um feedback visual da placa FPGA e de eventuais periféricos conectados a ela. Os alunos podem em tempo real acompanhar eventuais saídas dos circuitos descritos na placa FPGA observando os estados dos leds e dos displays de sete segmentos da mesma.

\section{FUNCIONAMENTO DO LABEAD}

A integração entre a plataforma IoT e os ESPs é feita através de um programa específico para aplicações embarcadas da Plataforma Arduino (script .ino), já a integração entre a plataforma IoT e a CPU localizada na bancada remota é feita através de um script Python. Esses scripts realizam a comunicação com o Blynk, que é a plataforma IoT aqui utilizada. Ambos necessitam estar presentes na bancada remota para o projeto funcionar corretamente. Cabe ressaltar aqui que a função do LabEAD no contexto da disciplina de Laboratório Digital é permitir ao aluno ter acesso ao ciclo completo de projeto de um sistema digital abordado na disciplina. Parte-se do princípio que o aluno irá realizar a codificação do sistema digital utilizando uma linguagem de descrição de hardware em sua própria residência, sendo a infraestrutura aqui apresentada responsável pela compilação, carga e testes do sistema digital descrito na placa FPGA.

Além dos arquivos correspondentes aos scripts apresentados, mais dois arquivos são necessários para permitir a correta execução do projeto. São eles: QAR do projeto no Quartus, que consiste em um arquivo único com todos os recursos de um projeto de circuito digital, e um arquivo TXT contendo a designação da pinagem (a pinagem consiste no mapeamento de entradas e saídas do circuito projetado pelo aluno em pinos físicos da placa FPGA). Ambos podem ser baixados do Google Drive, através de funcionalidade inclusa no script Python, e o arquivo de designação da pinagem também pode ser criado dentro do próprio script Python.

\subsection{Opções do Menu do Script Python}

A Figura 3 ilustra o fluxo básico de execução do script, partindo-se do princípio que tanto o arquivo QAR quanto o arquivo TXT de designação de pinagem já estão localizados na bancada remota.

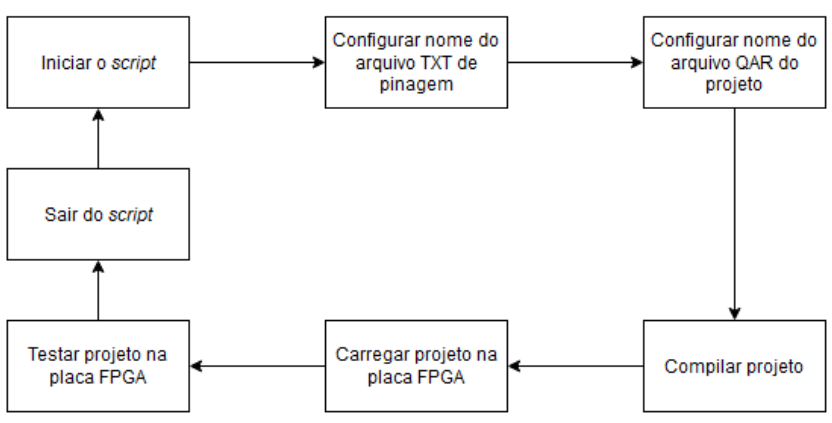

Figura 3: Fluxo básico de execução do script Python

Cada uma das etapas apresentada na figura ocorre mediante a um comando enviado no terminal do Blynk, pelo aluno em seu celular. A Tabela 1 apresenta cada comando juntamente com a sua abreviatura e função correspondente.

A seguir é realizada uma descrição completa do funcionamento de cada um dos comandos implementados.

Comando carregar (ca)

Este comando tem a função de carregar um arquivo executável da placa FPGA (arquivo SOF) na mesma. Ele só pode ser executado após a execução do comando de compilar o projeto. Ele foi implementado com um mecanismo de "tolerância a erros" de tal forma que caso um aluno tente fazer uma designação de pinos não permitida, o processo será finalizado e uma mensagem de erro será apresentada.

\section{Comando compilar (co)}

Este comando tem a função de compilar um projeto utilizando o Quartus Prime, através de chamadas pela linha de comando. Ele só pode ser executado após tanto o nome da experiência quanto o do projeto terem sido configurados. Sua execução consiste em extrair os arquivos presentes no QAR em uma nova pasta, limpar qualquer mapeamento de pinos feita previamente, utilizar o mapeamento presente no arquivo TXT de designação de pinos e compilar o projeto do sistema digital.

\section{Comando testar $(t)$}

Este comando tem a função de testar um projeto carregado previamente na placa FPGA. Para tal, ele realiza a troca de token no script .ino seguido de sua compilação e carga no ESP8266 com o Arduino CLI (command line interface). Esse processo ocorre apenas na primeira vez que o aluno utiliza o script após sua inicialização, não sendo repetido enquanto ele não sair do script. Logo após é possível 


\begin{tabular}{|c|c|l|}
\hline comando & abreviatura & \multicolumn{1}{c|}{ função } \\
\hline carregar & ca & carregar um projeto na placa FPGA \\
\hline compilar & co & compilar um projeto QAR com Intel Quartus Prime \\
\hline testar & $\mathrm{t}$ & alterar os valores de entrada \\
\hline pinar & $\mathrm{pi}$ & montar um arquivo de pinagem \\
\hline exp & $\mathrm{e}$ & mudar o nome da experiência (arquivo TXT de pinagem) \\
\hline projeto & $\mathrm{pr}$ & mudar o nome do projeto (arquivo QAR) \\
\hline baixar & $\mathrm{b}$ & baixar um arquivo do Google Drive \\
\hline mostrar & $\mathrm{m}$ & mostrar o valor atual dos parâmetros de exp/projeto \\
\hline arquivos & $\mathrm{ar}$ & mostrar os arquivos/diretórios \\
\hline remover & $\mathrm{r}$ & remover um arquivo/diretório \\
\hline ajudar & $\mathrm{a}$ & mostrar novamente o menu do script \\
\hline sair & $\mathrm{sa}$ & sair do script \\
\hline
\end{tabular}

Tabela 1: Comandos do script Python do LabEAD

alterar os valores de entrada da placa FPGA seja por comandos no terminal ou então por botões mapeados em pinos virtuais do Blynk.

\section{Comando mapear pinos (pi)}

Este comando tem a função de criar um arquivo TXT de designação de mapeamento de pinos dentro do próprio script. O aluno pode escolher um nome para o arquivo TXT e em seguida fornecer dados correspondentes às entradas/saídas do sistema digital e a pinagem desejada.

\section{Comando experiência (e)}

Este comando tem como função alterar o parâmetro correspondente ao nome da experiência, utilizando pelo script. Cada vez que esse comando é executado o aluno precisará compilar novamente o projeto antes de poder realizar sua carga na placa FPGA.

\section{Comando projeto (pr)}

Este comando tem como função alterar o parâmetro correspondente ao nome do projeto, utilizado pelo script. Cada vez que esse comando é executado o aluno precisará compilar novamente o projeto antes de poder realizar sua carga na placa FPGA

\section{Comando baixar (b)}

Este comando tem como função baixar um arquivo disponível no Google Drive do aluno. Para tal é necessário que o aluno disponibilize o arquivo em seu drive com a opção "Qualquer um que possua o link de acesso”. Será necessário escrever o ID do arquivo, que é um dos campos do link de compartilhamento do mesmo.

Comando mostrar (m)

Este comando tem como função mostrar ao aluno os valores atuais dos parâmetros correspondentes ao nome da experiência e ao nome do projeto.

Comando arquivos (ar)

Este comando tem como função permitir aos alunos a visualização dos arquivos/diretórios presentes na sua pasta da bancada remota. Os arquivos correspondentes aos scripts (Python e .ino) não são apresentados por se tratar de arquivos reservados.

\section{Comando remover $(\mathbf{r})$}

Este comando tem como função permitir ao aluno remover remotamente arquivos/diretórios presentes na sua pasta da bancada remota. Os arquivos correspondentes aos scripts (Python e .ino) não são apresentados por se tratar de arquivos reservados.

Comando ajuda (a)

Este comando tem como função mostrar novamente o menu.

\section{Comando sair (s)}

Este comando tem como função sair do script.

\subsection{Funcionamento do Script Arduino}

O script Arduino é uma descrição em linguagem de alto nível do código que controla o ESP8266, e é o responsável pela interação e monitoramento em tempo real com o projeto carregado na placa FPGA. Foi desenvolvido em Arduino IDE, com o uso da biblioteca Blynk (serviço de computação em nuvem para Internet das Coisas), biblioteca Software Serial (para comunicação serial UART), biblioteca Wire (comunicação com módulo expansor PCF8574), e biblioteca específica de comunicação WiFi para a placa de desenvolvimento Wemos D1 mini, que possui como base o ESP8266.

É importante destacar que os módulos expansores de portas PCF8574 foram necessários para que o número de entradas/saídas não ficasse limitado ao número disponível da placa Wemos D1 mini. Para que experimentos que necessitam de mais sinais de entrada e saída sejam realizados, como de transmissão e recepção serial (8 bits), duas placas expansoras com 8 pinos cada foram adicionadas a cada bancada remota, sendo controladas por meio de comunicação serial da biblioteca Wire.

Com o script Arduino, é possível por linha de comando controlar e monitorar pinos conectados aos pinos de entrada/saída da FPGA, por meio de um terminal virtual no aplicativo mobile Blynk, mapeado no pino virtual V0. O aplicativo do aluno deve ser configurado conforme Figura 4.

O controle de um pino físico do ESP8266 (D4, que é o led embutido na placa Wemos) está ilustrada na Figura 5. Através do comando "D4 1", o usuário comanda o pino D4 do ESP8266 para o nível alto. Com o comando "D4 0", o usuário comanda o pino D4 para nível baixo.

Destaca-se que o led embutido na placa Wemos D1 mini é ativo em baixo, e que, apesar do propósito da placa ESP8266 ser usada como ferramenta de interação com a FPGA, o controle de led do ESP8266 pelo celular foi utilizado para uma familiarização inicial com a interface mobile. 


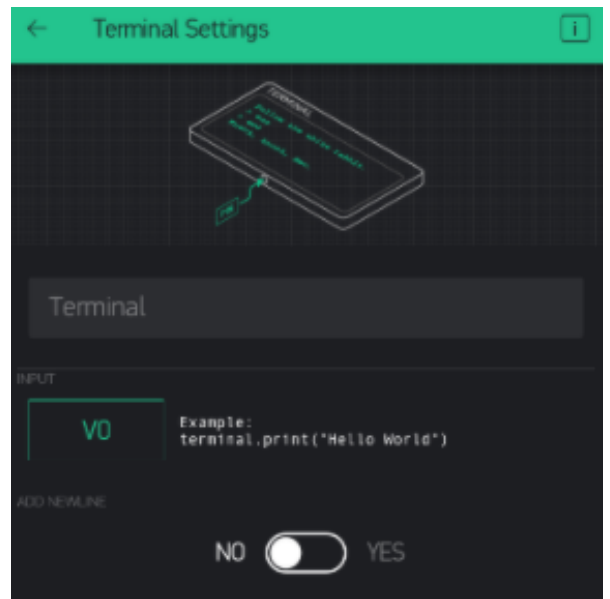

Figura 4: Configuração do terminal virtual no aplicativo

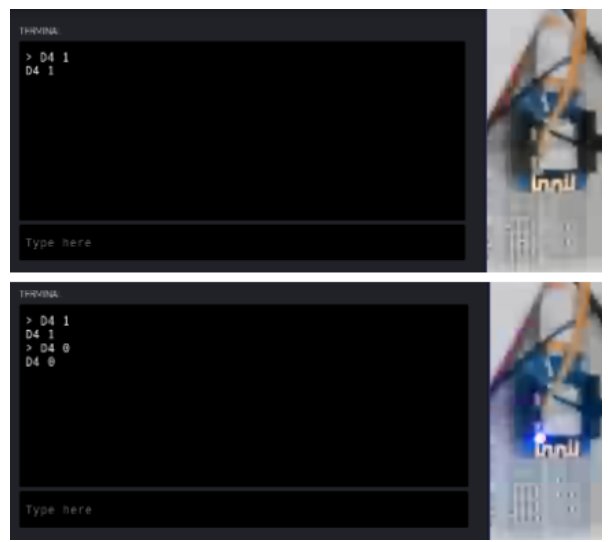

Figura 5: Controle do led embutido na placa Wemos pelo aplicativo mobile

O monitoramento está exemplificado na Figura 6. A partir do nome do pino, o script Arduino realiza a leitura deste pino e retorna o resultado no próprio terminal virtual V0.

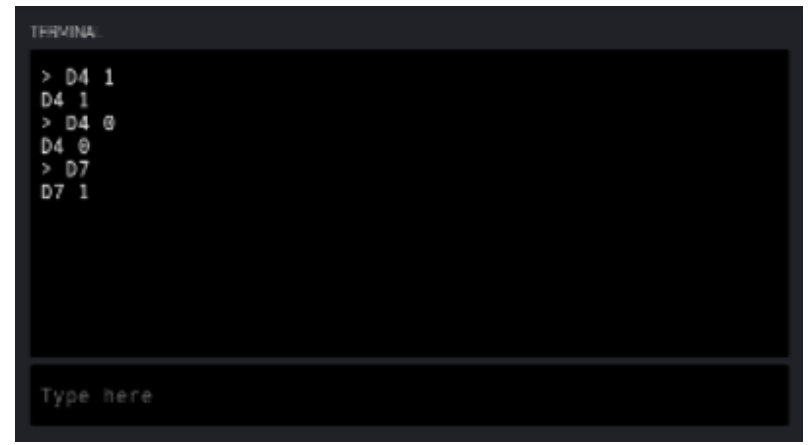

Figura 6: Monitoramento de entrada digital da placa Wemos pelo aplicativo mobile
Os pinos de entrada/saída da FPGA (GPIO) estão conectados diretamente nos pinos D5 e D6 do ESP8266 pois são responsáveis pela comunicação serial entre ambas as partes, enquanto diversos outros pinos estão conectados nos pinos de duas placas expansoras PCF8574.

Através da biblioteca Wire que implementa o protocolo I2C, o ESP8266 controla os 16 pinos dos 2 módulos expansores, uma vez que um módulo expansor está no endereço 0 e outro está no endereço 1 (a configuração de endereços é realizada através de jumpers nos módulos PCF8574).

A integração com a biblioteca Blynk permite o mapeamento dos pinos físicos (do próprio ESP8266 e dos expansores) em pinos virtuais. Por sua vez, estes pinos virtuais podem ser mapeados em botões e leds virtuais no aplicativo Blynk.

Desta forma, os pinos da placa expansora podem ser comandados a partir do terminal virtual V0, considerando o mapeamento de E0 a E11, ou então por meio de pinos virtuais mapeados em widgets no aplicativo mobile Blynk.

Espera-se que as duas possibilidades de interação tornem possível uma maior liberdade na escolha de interfaces personalizadas por experimento, e tornem possível a execução de todos os experimentos com os créditos do nível gratuito da conta do Blynk.

A Figura 7 mostra o mapeamento nos pinos do terminal virtual $\mathrm{V} 0$ e nos demais pinos virtuais.

\section{MÉTODO}

Nesta seção são descritas as atividades realizadas com a plataforma, tanto no contexto do workshop com os docentes do departamento quanto no contexto da disciplina de laboratório de eletrônica digital.

\subsection{Workshop Docente}

O Workshop com os docentes foi uma oficina realizada no modelo hands-on no dia 14/08/2020. Neste evento, os docentes tiveram a oportunidade de interagir diretamente com a plataforma desenvolvida. A importância deste evento está no fato que a plataforma aqui apresentada foi a única desenvolvida pelo departamento no ano corrente para contornar o desafio do aprendizado prático respeitando os critérios de distanciamento social.

Durante a oficina foi possível apresentar em um espaço de tempo de 3 horas a motivação, o desenvolvimento e os desafios deste tipo de projeto, o que serviu de motivação para os docentes pensarem em alternativas para as suas disciplinas teóricas e práticas. Intercalando explicações com momentos hands-on, os participantes puderam assumir o papel do aluno da disciplina, interagindo com a plataforma e realizando os passos de compilação, carga e testes de um projeto digital.

Ao final do workshop do dia 14/08/2020, foi aplicado um questionário para colher as percepções dos docentes sobre o oferecimento remoto da disciplina. Os resultados deste questionário serão apresentados em seção posterior.

\subsection{Aulas Realizadas}

A disciplina de laboratório de eletrônica digital possui seu oferecimento remoto síncrono, ou seja, em horário definido estabelecido para a disciplina, utilizando para tal salas no Google Meets. A plataforma do LabEAD suportou a execução de 6 experimentos de forma 


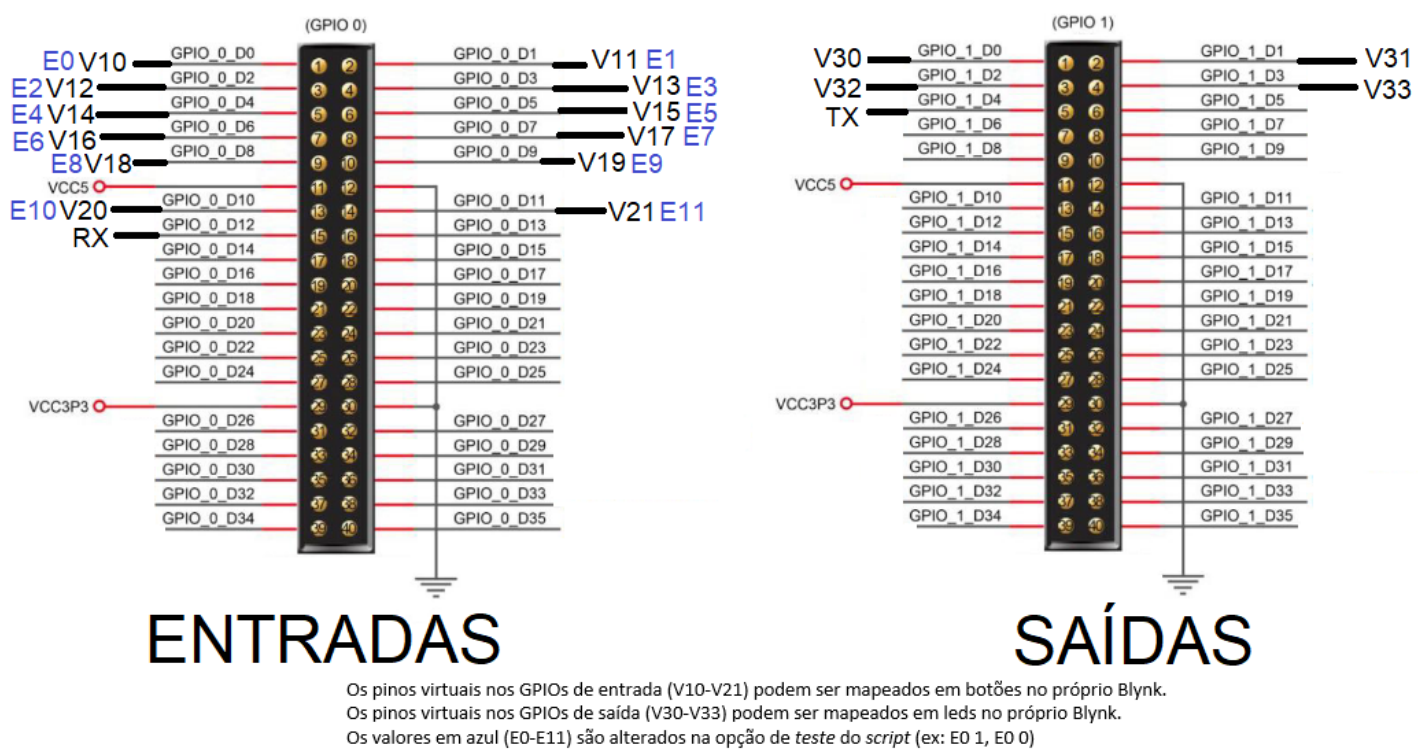

Figura 7: Esquema de montagem de um kit LabEAD no Laboratório Digital

remota entre $01 / 09 / 2020$ e $07 / 10 / 2020$, em sessões síncronas por 4 docentes e 32 alunos.

No começo da aula os docentes, e os discentes se encontram na sala principal e em um primeiro momento os alunos realizam uma prova dissertativa cuja submissão é realizada em um ambiente virtual de aprendizagem. O objetivo desta prova é avaliar o entendimento do aluno sobre a experiência a ser realizada, o que deve ser consequência direta da qualidade do planejamento realizado para a mesma. Em seguida, é realizada uma explicação sobre a experiência do dia, onde os docentes realizam uma breve apresentação acerca da fundamentação teórica que tange a experiência além de fornecer dicas de testes e depuração sobre o circuito digital desenvolvido naquela semana. Os pesquisadores então apresentam aspectos técnicos da plataforma que serão utilizados durante a aula. Após o término da explicação, os alunos saem da sala principal do Google Meets e vão para suas salas de bancada (que representam uma analogia com a bancada física do laboratório) e tem o restante da aula para demonstrar o funcionamento do seu circuito digital, além de realizar testes e depurações nos casos onde os circuitos não funcionaram corretamente. Neste momento eles contam com o suporte dos docentes, pesquisadores e técnicos para resolver auxiliar na resolução de eventuais problemas que venham a surgir.

Ao decorrer das aulas da disciplina os alunos foram tendo contato com novas funcionalidades da plataforma, resultado em uma curva de aprendizado e de maturidade dos discentes. Ressalta-se aqui um esforço grande por parte da equipe em introduzir estas novas funcionalidades de maneira gradual e tênue, visando minimizar a ansiedade da turma. Observou-se uma evolução e um conforto dos discentes ao decorrer das aulas em relação ao novo modelo de aulas práticas remotas aqui estabelecidas.

Na próxima seção, serão apresentados os resultados do questionário realizado com os alunos logo após o término do primeiro experimento (01/09/2020), após o primeiro contato dos mesmos com a plataforma. Em seguida, será feita uma discussão e uma análise crítica sobre a evolução do desempenho dos discentes com o uso da plataforma após o sexto experimento (07/10/2020).

\section{RESULTADOS}

Nesta seção são apresentados os resultados dos dois questionários aplicados (com os docentes e com os discentes), além de uma análise crítica sobre a interação dos alunos com a plataforma em questão.

\subsection{Workshop com Docentes}

Houve a participação de 10 docentes não relacionados ao oferecimento da disciplina em questão no workshop realizado no dia 14/08/2020, e 8 docentes responderam ao questionário.

A Figura 8 mostra o grau de familiaridade dos docentes com a disciplina de laboratório de eletrônica digital. Somente 1 desses 8 docentes possuía menor familiaridade com a disciplina, sendo que mais da metade considerou que possui alto grau de familiaridade.

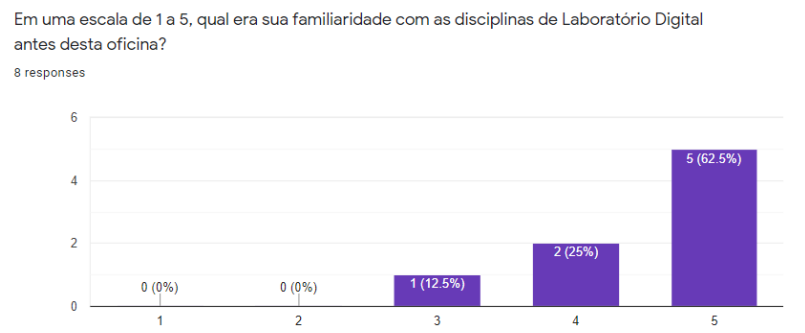

Figura 8: Resultado da questão 1 com docentes

Conforme mostra a Figura 9, o workshop foi avaliado de forma positiva pelos docentes participantes. De um total de 10 participantes da oficina, 2 não responderam ao questionário, enquanto 
2 avaliaram a oficina com nota 4, e 6 docentes atribuíram nota máxima.

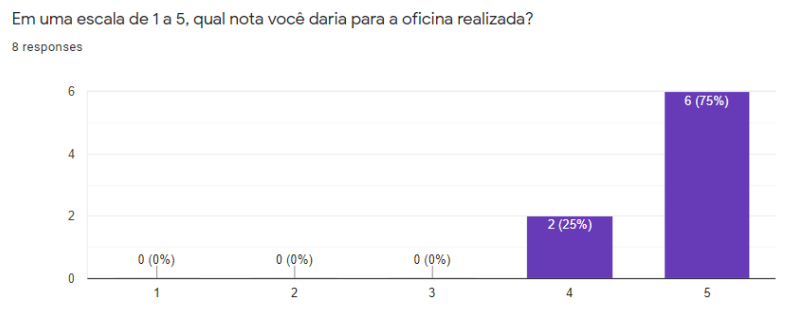

Figura 9: Resultado da questão 2 com docentes

A seção de comentários gerais sobre as notas permitiu aos docentes que participaram do workshop expressarem sua opinião de forma qualitativa. Os cinco comentários a seguir caracterizam opiniões positivas sobre a plataforma apresentada: "Gostei muito! A nota foi pois achei que será um sucesso durante as aulas"; "Excelente conteudo"; "Experiência excelente."; "parabens pelo trabalho e solucao para esse ambiente de pandemia."; "A oficina foi muito boa, permitiu que cada participante fizesse um hands on das funcionalidades do LabEAD e desmistificasse a ideia de conexão remota da placa.”.

Os três comentários abaixo destacam pontos a melhorar.

O primeiro comentário destaca uma dificuldade de visualização por parte de um docente: "Acho que devia mostrar mais a tela, para todos poderem ver o que está acontecendo ou vai acontecendo".

Outro docente sugeriu que oficinas futuras fossem realizadas em grupos menores: "Parabéns pela iniciativa. Como sugestão para eventos futuros seria a constituição de grupos menores.".

Por fim, um docente enfatizou o uso de mecanismos de segurança para o acesso à plataforma: "Upload do QAR, uso de https".

\subsection{Questionário Discente}

Nesta seção, é apresentado o resultado do questionário após a experiência inicial com os alunos nos dias 01/09/2020 e 02/09/2020. Dos 32 alunos, 25 responderam ao questionário.

A primeira pergunta tem o objetivo de levantar o nível de conhecimento prévio dos alunos em relação a conteúdos abordados no curso: comunicação digital serial, sensores e atuadores. $40 \%$ dos alunos concordaram que conhecem o aspecto teórico, e $60 \%$ consideram que não possuem conhecimento sobre estes conceitos.

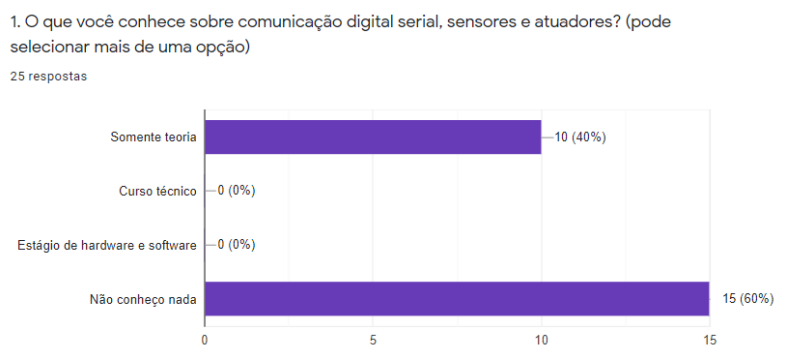

Figura 10: Resultado da questão 1 com alunos
A seção de complemento da pergunta 1 permitiu aos alunos expressarem como adquiriram algum conhecimento prévio: "Conheço muito pouco, apenas o conteudo abordado em disciplinas anteriores do curso"; "Projetos pessoais"; "Conhecimentos básicos vistos nas disciplinas de Sistemas DIgitais".

A segunda pergunta disserta sobre aulas remotas. $60 \%$ dos alunos alegam ter feito cursos teóricos de forma remota, e 56\% dizem ter realizado reuniões e simulações de forma remota. Observa-se que apenas um aluno fez curso prático remotamente.

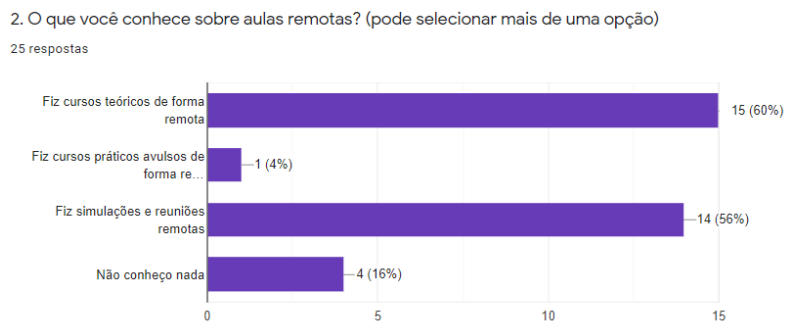

Figura 11: Resultado da questão 2 com alunos

O complemento da pergunta 2 demonstra comentários sobre o ensino à distância: "Tivemos EAD no primeiro quadri." "Já trabalhei e fiz alguns cursos remotos, mas nunca na graduação"; "Fiz alguns cursos com aulas remotas durante a pandemia e com isso tive um pouco a noção do que é ter aulas remotas.".

Um comentário destaca como o único aluno fez atividades de laboratório de forma remota: "Aulas teóricas feitas remotamente e algumas atividades de laboratório". Isto pode estar relacionado a alguma disciplina optativa realizada pelo aluno em questão.

A terceira pergunta aborda a percepção dos alunos sobre a primeira parte do experimento, no qual os alunos interagiram com o módulo ESP8266 instalado no laboratório, e controlar um led integrado a esta placa, e visualizar seu resultado com uma webcam e videoconferência no computador do laboratório.

Mais de 3/4 dos alunos entrevistados entenderam o acesso remoto e gostaram da oportunidade de conexão entre componentes suportada pela plataforma, conforme Figura 12. Somente um aluno não gostou da proposta.

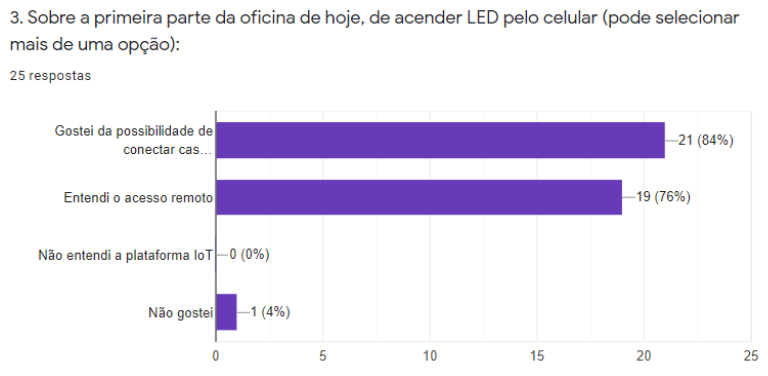

Figura 12: Resultado da questão 3 com alunos

Os comentários a seguir ilustram a percepção positiva dos discentes: "Gostaria de parabenizar a equipe docente pela atividade 
preparada para a aula. Foi a melhor experiência de aulas remotas que tive até o momento, mesmo com uma série de complicações (que foram rapidamente resolvidas pelo professor XXX)"; "ISSO FOI INCRIVVEL PARABENS PROS PROFESSORES ORGULHO DE SER ALUNO DE VOCES !!! (O texto em caixa alta é pq eu to muito animado, parabéns mesmo, professores)."; "Achei tudo muito bom, parabéns aos envolvidos.".

O aluno que não gostou da proposta forneceu alguns motivos para sua opinião, como falha no suporte e configuração do computador de sua bancada: "Meu computador remoto desligou no meio da experiência, e nem o XXX nem XXX conseguiram ajudar. $\mathrm{O}$ blynk.ino estava configurado incorretamente, também. Não consegui fazer nem a Atividade 1 nem a 2.”. É importante destacar que há sempre um técnico no laboratório para prestar auxílio às atividades remotas.

A segunda parte da oficina sobre o uso do terminal virtual pelo celular para compilação, carregamento e interação com o circuito digital obteve um resultado positivo: $88 \%$ dos alunos alegaram ter gostado da proposta, conforme Figura 13.

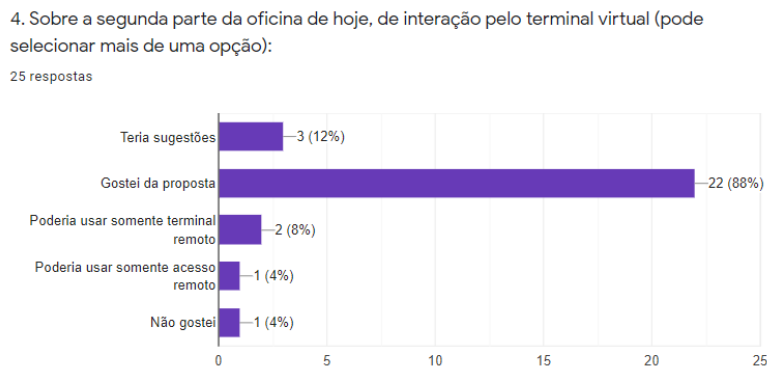

Figura 13: Resultado da questão 4 com alunos

Os alunos expressaram pontos a melhorar em relação aos $f e-$ edbacks fornecidos durante o processo: "Achei confusa a saida do terminal do prompt do computador"; "Senti falta de logs mais específicos no terminal remoto, mas isso foi sanado pelo acesso remoto ao terminal da máquina utilizada"; "Não apareceu as atualizações no terminal, apenas "O aluno está utilizando o script". Além disso, enquanto compila, não temos nenhum feedback da porcentagem de compilação, o que é problemático pois parece que o programa travou."; "Houve pequenos problemas de configuração (que puderam ser resolvidos de maneira tranquila). Houve uma pequena demora na execução dos comandos do terminal, o que gerou dúvidas quanto ao fato de os comandos terem sido corretamente executados.”.

Um aluno destacou sua preferência por botões virtuais em detrimento de interações pelo terminal: "Achei legal a proposta de mexer na FPGA pelo terminal, foi uma boa experiência, mas acho que ainda prefiro ter os botões/switches virtuais.”.

Sobre a proposta de uso da plataforma desenvolvida em um projeto dirigido a ser realizado pelos alunos na segunda parte do curso, $76 \%$ dos alunos afirmam terem gostado do projeto. $24 \%$ dos alunos preferem aulas dirigidas.

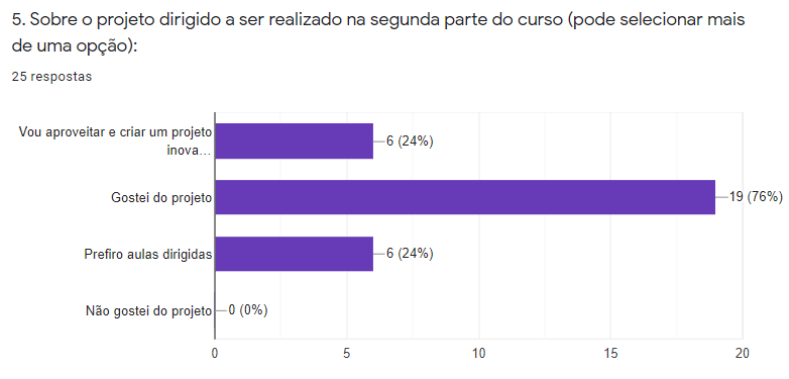

Figura 14: Resultado da questão 5 com alunos

Complemento da pergunta 5:

O comentário a seguir fornece a opinião de um aluno que gostou da proposta, devido à flexibilidade de propor e executar um projeto de interesse do aluno: "Ainda não tenho uma ideia clara do que farei, mas gosto de ter a abertura de poder desenvolver um projeto criado por mim e minha dupla”.

Outro comentário expressa a insegurança do aluno em realizar o projeto dirigido durante o período de distanciamento social: "Apesar de preferir aulas dirigidas (por poderem ser feitas de maneira mais sistemática), a ideia de um projeto é interessante. No entanto, devem ser avaliadas as dificuldades frente o distanciamento social e o acesso remoto."

\subsection{Considerações sobre o andamento da disciplina}

A introdução de uma plataforma inédita para viabilizar o oferecimento da disciplina em caráter remoto traz consigo um desafio adicional, que é o de diminuir a ansiedade dos alunos. Toda mudança traz consigo algum estranhamento e/ou desconforto e no contexto desta disciplina, o medo de falhar é algo que permeia o imaginário dos alunos. Este medo pode estar relacionado a vivências anteriores dos discentes, onde o sucesso é decorrente de uma situação onde tudo funcione conforme o esperados, sem problemas no projeto, sendo que o mesmo nem sempre ocorre no contexto desta disciplina.

Desta forma, a equipe teve que trabalhar o fator ansiedade com os alunos desde a primeira aula, expondo as particularidades do oferecimento remoto da disciplina e mantendo um canal de comunicação sólido com eles. Esta comunicação foi responsável por uma série de modificações realizadas na plataforma ao decorrer da disciplina, visando acatar pedidos feitos para melhorar o uso da plataforma.

Além disso, foi montado um FAQ baseado nos questionamentos levantados durante a aula e nos erros encontrados durante a interação do aluno com a plataforma. O fato do aluno entender o seu reconhecimento e importância pela equipe nesta comunicação também atua como aspecto motivacional para o seu bom desempenho nas experiências da disciplina, mesmo com os desafios de seu oferecimento remoto. Ressalta-se também aqui a importância dos técnicos de laboratório, que viabilizam um diálogo mais informal e próximo da realidade do aluno, o que ajuda a reduzir sua ansiedade. 


\section{CONSIDERAÇÕES FINAIS}

Este artigo apresentou as percepções docentes e discentes sobre a plataforma desenvolvida com o intuito de viabilizar o oferecimento remoto de uma disciplina de laboratório de eletrônica digital. Enxerga-se aqui uma grande oportunidade de expansão, de tal forma a ampliar o escopo da plataforma para viabilizar também outras disciplinas correlatas. Os índices elevados de aceitação atrelados ao retorno positivo por parte dos alunos apontam que este novo modo de ensino não precisa necessariamente ser algo limitado ao contexto atual da pandemia, mas sim pode ser aplicado no futuro "novo normal", em um sistema de ensino híbrido.

A integração com o homelab e o andamento dos projetos propostos pelos alunos na disciplina são apresentados como oportunidades de trabalhos futuros mais imediatos. Além disso, ressalta-se também a necessidade de uma avaliação mais metodológica para comparar o aprendizado obtido neste oferecimento em relação ao obtido em um oferecimento presencial.

\section{AGRADECIMENTOS}

Agradecemos ao fundo patrimonial Amigos da Poli pelo apoio.

\section{REFERÊNCIAS}

[1] Daniela Melaré Vieira Barros. 2009. Estilos de uso do espaço virtual: Como se aprende e se ensina no virtual? Revista Inter-ação (2009), 51-74.

[2] Blynk. 2020. Blynk. https://blynk.io/. Acesso: 10/07/2020.

[3] JA Del Alamo. 2007. MIT iLabs: Towards a community of internet accessible laboratories. In International conference on remote engineering and virtual instrumentation.

[4] Jinalkumar Doshi, Pratiksha Patil, Zalak Dave, Ganesh Gore, Jonathan Joshi, Reena Sonkusare, and Surendra Rathod. 2015. Implementing a cloud based xilinx ise fpga design platform for integrated remote labs. In 2015 International Conference on Advances in Computing, Communications and Informatics (ICACCI) IEEE, 533-537.

[5] Intel. 2020. Altera DE0 FPGA Board. https://www.intel.com/content/www/us/ en/programmable/solutions/partners/partner-profile/terasic-inc-/board/alterade0-board.html. Acesso: 10/07/2020.

[6] Jasveer Singh T Jethra, Sachin B Patkar, and Shamik Datta. 2014. Remote triggered fpga based automated system. In 2014 11th International Conference on Remote Engineering and Virtual Instrumentation (REV). IEEE, 309-314.

[7] David Lowe, Steve Murray, Euan Lindsay, and Dikai Liu. 2009. Evolving remote laboratory architectures to leverage emerging internet technologies. IEEE Transactions on learning technologies 2, 4 (2009), 289-294.

[8] Abd El-Rahman Mohsen, Mohamed Youssef GadAlrab, Zeina elhaya Mahmoud Gameel Alshaer, Mahmoud Asy, and Hassan Mostafa. 2019. Remote FPGA Lab For ZYNQ and Virtex-7 Kits. In 2019 IEEE 62nd International Midwest Symposium on Circuits and Systems (MWSCAS). IEEE, 185-188.

[9] F. Morgan, S. Cawley, M. Kane, A. Coffey, and F. Callaly. 2014. Remote FPGA Lab applications, interactive timing diagrams and assessment. In 25th IET Irish Signals Systems Conference 2014 and 2014 China-Ireland International Conference on Information and Communications Technologies (ISSC 2014/CIICT 2014). 221-226.

[10] Oeit. 2020. iLabs. http://icampus.mit.edu/projects/ilabs/. Acesso em 30 de julho de 2020.

[11] Andrea Schwandt and Marco Winzker. 2019. Make it open-Improving usability and availability of an FPGA remote lab. In 2019 IEEE Global Engineering Education Conference (EDUCON). IEEE, 232-236.

[12] James Trevelyan. 2004. Lessons learned from 10 years experience with remote laboratories. In International Conference on Engineering Education and Research, Vol. 11. 2007.

[13] Marco Winzker, Roberto Kiessling, Andrea Schwandt, Carlos Sosa Paez, and Shatha Abu Shanab. 2018. Teaching across the ocean with video lectures and remote-lab. In 2018 IEEE World Engineering Education Conference (EDUNINE) IEEE, 1-4.

\section{A QUESTIONÁRIOS REALIZADOS}

\section{A.1 Questionário após oficina com docentes}

Formulário para avaliação e envio de feedbacks da oficina do LabEAD realizada em 14/08/2020.

- Endereço de email (obrigatório)

- Nome completo (obrigatório)

- Em uma escala de 1 a 5, qual era sua familiaridade com as disciplinas de Laboratório Digital antes desta oficina? (Nota de 1 a 5, Obrigatório)

- Em uma escala de 1 a 5 , qual nota você daria para a oficina realizada? (Nota de 1 a 5, Obrigatório)

- Gostaria de fazer comentários gerais sobre a nota?

- Para nos ajudar a melhorar, por favor descreva aqui sugestões para a equipe.

\section{A.2 Questionário após oficina com alunos}

Formulário para avaliação e envio de feedbacks da oficina do LabEAD realizada em 01/09/2020 e 02/09/2020.

Cada pergunta possui espaço para inclusão de considerações e comentários.

(1) O que você conhece sobre comunicação digital serial, sensores e atuadores? (pode selecionar mais de uma opção)

- Somente teoria

- Curso técnico

- Estágio de hardware e software

- Não conheço nada

(2) O que você conhece sobre aulas remotas? (pode selecionar mais de uma opção)

- Fiz cursos teóricos de forma remota

- Fiz cursos práticos avulsos de forma remota

- Fiz simulações e reuniões remotas

- Não conheço nada

(3) Sobre a primeira parte da oficina de hoje, de acender LED pelo celular (pode selecionar mais de uma opção):

- Gostei da possibilidade de conectar casa e escola

- Entendi o acesso remoto

- Não entendi a plataforma IoT

- Não gostei

(4) Sobre a segunda parte da oficina de hoje, de interação pelo terminal virtual (pode selecionar mais de uma opção):

- Teria sugestões

- Gostei da proposta

- Poderia usar somente terminal remoto

- Poderia usar somente acesso remoto

- Não gostei

(5) Sobre o projeto dirigido a ser realizado na segunda parte do curso (pode selecionar mais de uma opção):

- Vou aproveitar e criar um projeto inovador

- Gostei do projeto

- Prefiro aulas dirigidas

- Não gostei do projeto 